BULL. AUSTRAL. MATH . SOC.

VOL. 29(1984), 129-135.

\title{
A CHARACTERIZATION OF WEAK PEAK SETS \\ FOR FUNCTION ALGEBRAS
}

\author{
KRZYSZTOF JAROSZ
}

Let $A \subset C(S)$ be a function algebra. In this paper we prove that $S_{0}=\bar{S}_{0} \subset S$ is a weak peak set for $A$ if and only if for any open neighbourhood $U$ of $S_{O}$ there is an $f$ in $A$ such that $\|f\| \leqslant 2,|f(x)-1| \leqslant 1 / 3$ on $S_{0}$ and $|f(x)| \leqslant 1 / 3$ on $S \backslash U$.

Let $S$ be a compact Hausdorff space. We shall denote by $C(S)$ $\left(C_{R}(S)\right)$ the Banach algebra of all complex (real) valued continuous functions on $S$, provided with a usual supremum norm. Let $A$ be a function algebra on $S$, i.e. a closed subalgebra of $C(S)$ which contains the constant functions and which separates points of $S$. A closed subset $S_{0}$ of $S$ is called a weak peak set for $A$ if for any open neighbourhood $U$ of $S_{0}$ there is an $f$ in $A$ such that $1=\|f\|=f(s)$ for $s \in S_{0}$ and $|f(s)|<1$ for $s \in S \backslash U$. A one-point weak peak set is called a weak peak point. A classical Bishop's the so-called "1/4 - 3/4" criterion (see e.g. [4] p. 263) gives the following characterization of weak peak points :

THEOREM [Bishop]. Let $A$ be a function algebra on $S$. A point $s_{0} \in S$ is a weak peak point for $A$ if and only if there are constants $K \geqslant 1$ and $c<1$ such that for any open neighbourhood $U$ of $s_{0}$ there is an $f$ in $A$ such that $\|f\| \leqslant K, f\left(s_{o}\right)=1$ and $|f(e)| \leqslant c$ for $s \in S-U$.

Received 24 February 1984.

Copyright Clearance Centre, Inc. Serial-fee code: 0004-9727/84 $\$ A 2.00+0.00$ 
The aim of this note is to give the following generalization of the above criterion:

THEOREM. Let $A$ be a function algebra on a compact Hausdorff space $S$ and let $S_{0}$ be a closed subset of $S$. Then $S_{0}$ is a weak peak set for $A$ if and only if there are constants $k \geqslant 1, c_{1} \geqslant 0, c_{2} \geqslant 0$ with $c_{1}+c_{2}<1$ such that for any open neighbourhood $U$ of $S_{0}$ there is an $f$ in $A$ such that $\|f\| \leqslant K,|f(s)-1| \leqslant c_{2}$ for $s \in S_{0}$ and $|f(s)| \leqslant c_{1}$ for $s \in S>U$.

Please note that the definition of a weak peak set is evidently equivalent to the following one ([4]):

Let $A$ be a function algebra on $S$ and let $S_{O}$ be a closed subset of $S$ then $S_{0}$ is a weak peak set for $A$ if and only if for any $\varepsilon>0$ and any open neighbourhood $U$ of $S_{O}$ there is an $f$ in $A$ such that $1=\|f\|=f(s)$ for $s \in S_{O}$ and $|f(s)|<\varepsilon$ for $s \in S \backslash U$.

Hence the "only if" part of our theorem is trivial: that is, if $S_{0} \subset S$ is a weak peak set for $A$ then we can take $c_{1}, c_{2}$ any positive numbers and put $K=1$.

We divide the proof of our theorem into four steps; the proof of the second step is based on Bishop's proof of his criterion.

Assume that the assumption of Theorem is fulfilled.

STEP 1. For any $\varepsilon>0$ there is a positive constant $K(\varepsilon)$ such that for any open neighbourhood $U$ of $S_{0}$ there is an $f$ in $A$ such that $|f(s)| \leqslant \varepsilon$ for $s \in S \backslash U ;|f(s)| \leqslant 1$ and $|f(s)-1| \leqslant \varepsilon$ for $s \in S_{o}$ and $\|f\| \leqslant K(\varepsilon)$.

Proof. Let $f_{1} \in A$ be such that $\left|f_{1}(s)\right| \leqslant c_{1}$ for $s \in S \backslash U$, $\left|f_{1}(s)-1\right| \leqslant c_{2}$ for $s \in S_{0}$ and $\left\|f_{1}\right\| \leqslant K$. Since the discs $D_{1}=\left\{z \in \Gamma:|z| \leqslant c_{1}\right\}$ and $D_{2}=\left\{z \in \Gamma:|z-1| \leqslant c_{2}\right\}$ are disjoint then, by Runge Theorem, there is a polynomial $p$ such that $p(z) \leqslant \varepsilon$ for $z \in D_{1}$ and $\left|p(z)-\left(1-\frac{\varepsilon}{2}\right)\right| \leqslant \frac{\varepsilon}{2}$ for $z \in D_{2}$. 
Put $K(\varepsilon)=\sup \{|p(z)|:|z| \leqslant K\}$ and $f=p \circ f_{1} \in A$.

STEP 2. Assume that there are constants $K_{1}$ and $\varepsilon<1$ such that for any open neighbourhood $U$ of $S_{o}$ there is an $f$ in $A$ such that $|f(s)| \leqslant \varepsilon$ for $s \in S-U ;|f(s)| \leqslant 1$ and $|f(s)-1| \leqslant \varepsilon$ for $s \in S_{0}$ and $\|f\| \leqslant K_{1}$, then for any open neighbourhood $U$ of $S_{0}$ there is a $g$ in $A$ such that $|g(s)| \leqslant \varepsilon$ for $s \in S \backslash U ;|g(s)-1| \leqslant \varepsilon$ for $s \in S_{O}$ and $\|g\| \leqslant 1$.

Proof. Fix any $x<1$ with

$$
a=\left(K_{1}-1\right)-x\left(K_{1}-\varepsilon\right)<0
$$

and a decreasing sequence of positive numbers $\varepsilon_{n}$ such that

$$
\varepsilon_{n}\left(1-x^{n}\right)+x^{n} a<0 \text { for } n \geqslant 1
$$

We define by induction a sequence of functions $\left(h_{n}\right)_{n=1}^{\infty}$ from $A$. Let $h_{1} \in A$ be any function from $A$ such that $\left|h_{1}(s)\right| \leqslant \varepsilon$ for $s \in S \backslash U$; $\left|h_{1}(s)\right| \leqslant 1$ and $\left|h_{1}(s)-1\right| \leqslant \varepsilon$ for $s \in S_{0}$ and $\left\|h_{1}\right\| \leqslant K_{1}$.

Assume we have defined $h_{1}, \ldots, h_{n}$ then put

$$
W_{n}=\left\{s: \max _{1 \leqslant j \leqslant n}\left|h_{j}(s)\right| \geqslant 1+\varepsilon_{n}\right\} .
$$

The set $W_{n}$ is a closed subset of $S \backslash S_{0}$ so there is an $h_{n+1} \in A$ such that

$$
\begin{aligned}
& \left|h_{n+1}(s)\right| \leqslant \varepsilon \text { for } s \in(S \backslash U) \cup W_{n} ; \\
& \left|h_{n+1}(s)\right| \leqslant 1 \text { and }\left|h_{n+1}(s)-1\right| \leqslant \varepsilon \text { for } s \in S_{0}
\end{aligned}
$$

and

$$
\left\|h_{n+1}\right\| \leqslant K_{1}
$$


Let

$$
g=(1-x) \sum_{j=1}^{\infty} x^{j-1} \cdot h_{j}
$$

We have evidently $|g(s)| \leqslant 1$ and $|g(s)-1| \leqslant \varepsilon$ for $s \in s_{O}$, $|g(s)| \leqslant \varepsilon$ for $s \in S \backslash U$, and $|g(s)| \leqslant 1$ for $s \in S \backslash \bigcup_{n=1}^{\infty} W_{n}$. It remains to show that if $s \in \bigcup_{n=1}^{\infty} W_{n}$ then $|g(s)| \leqslant 1$.

The sequence $\left(w_{n}\right)_{n=1}^{\infty}$ is an increasing sequence of compact sets so if $s \in \underset{n=1}{\bigcup} W_{n}$ then there is a positive integer $m$ such that $s \in W_{m+1}$ but $s \notin W_{m}$ for $m \geqslant 0$ (we put $W_{0}=\emptyset$ ).

We have

$$
\begin{aligned}
& \left|h_{j}(s)\right| \leqslant 1+\varepsilon_{m} \text { for } j \leqslant m, \\
& \left|h_{m+1}(s)\right| \leqslant K_{1}, \\
& \left|h_{j}(s)\right| \leqslant \varepsilon \text { for } j \geqslant m+2,
\end{aligned}
$$

hence

$$
\begin{aligned}
|g(s)| & \leqslant(1-x)\left(\left(1+\varepsilon_{m}\right) \sum_{j=1}^{m} x^{j-1}+K_{1} x^{m}+\varepsilon \sum_{j=m+2}^{\infty} x^{j-1}\right) \\
& =1+\varepsilon_{m}\left(1-x^{m}\right)+x^{m}\left(K_{1}-1-x\left(K_{1}-\varepsilon\right)\right)<1 .
\end{aligned}
$$

STEP 3. Let $M$ be a closed subspace of $C(S)$, let $\pi$ be a canonical map from $M$ onto $\left.{ }^{M}\right|_{S_{0}}=\left\{f_{\left.\right|_{S_{0}}}: f \in M\right\} \subset C\left(S_{0}\right) \pi(f)=f_{S_{0}}$, and let $\tilde{\pi}: M /$ ker $\left.\rightarrow{ }^{M}\right|_{S_{0}}: \tilde{\pi}(f+\operatorname{ker} \pi)=f_{\mid S_{0}}$. Then if $\tilde{\pi}$ is not an isometry then there are measures $\mu$ on $S_{0}$ and $\nu$ on $S-S_{0}$ such that $\mu-\nu \perp M$ but the measure $\mu$ represents a non zero functional on $M$. Proof. Assume $\tilde{\pi}$ is not an isometry, there is a functional $F_{0}$ on 


\section{Weak peak sets}

${ }^{M}{ }_{S_{0}}$ represented by a measure $\mu_{0}$ on $S_{0}$ such that $\left\|F_{0}\right\|=\operatorname{var}\left(\mu_{0}\right)=1$ but $\left\|\pi *\left(F_{0}\right)\right\|=t<1$. Let $\nu_{0}$ be any measure on $S$ which represents the functional $\pi *\left(F_{0}\right)$ with $\operatorname{var}\left(\nu_{0}\right)=t$. Since $\operatorname{var}\left(\left.\nu_{0}\right|_{S_{0}}\right) \leqslant t<1$ then the measure $\mu_{0}-v_{o} \mid S_{0}$ is not orthogonal to $M$ and we can put $\mu=\mu_{0}-v_{0 \mid S_{0}}, \quad v=v_{0} \mid S \backslash S_{0}$.

To end the proof of Theorem now fix an open neighbourhood $U$ of $S_{O}$ and let $q \in C_{R}(S)$ be such that

$$
q_{S_{0}} \equiv 1, q_{\mid S \backslash U} \equiv 3, \quad 1 \leqslant q \leqslant 3
$$

and put

$$
M=\{q \cdot g \in C(S): g \in A\} .
$$

We shall prove that $\tilde{\pi}: M /$ kerm $\rightarrow{ }^{M} S_{0}$ is an isometry. Assume the contrary and let $\mu, \nu$ be as in step 3. We can assume that the norm of $\mu$ on ${ }^{M} \mid S_{o}$ is equal to 2 and let $f \in M$ be such that $\int f d \mu=1$ and $\|f\|_{S_{0}} \leqslant 1$. Fix $\left.0<\delta<\frac{1}{2}\|f\|(1+\operatorname{var}(\nu))\right)^{-1}$. The regularity of the measure $v$ provides there is an open neighbourhood $V$ of $S_{0}$ such that $|v|(V)<\delta$ and, by steps 1 and 2 , there is an $f_{0}$ in $A$ such that $\left\|f_{O}\right\|_{S \backslash V} \leqslant \delta,\left\|f_{0}-1\right\|_{S_{0}} \leqslant \delta$ and $\left\|f_{0}\right\| \leqslant 1$. since $\nu-\mu \perp M$ and $f_{0} \in M$ we have

$$
\begin{aligned}
& \frac{1}{2}<1-2 \delta \leqslant\left|\int_{S_{0}} f d \mu\right|-\left|\int_{S_{0}} f\left(1-f_{O}\right) d \mu\right| \\
& \quad \leqslant\left|\int_{S_{0}} f f_{O} d \mu\right|=\left.\right|_{S} \int_{S_{0}} f f_{O} d \nu|\leqslant| \int_{V} f f_{O} d \nu|+|_{S} \int_{V} f f_{O} d \nu \mid \\
& \quad \leqslant \delta\|f\|+\operatorname{var}(\nu) \delta\|f\|<\frac{1}{2} .
\end{aligned}
$$


So we have proved that $\tilde{\pi}$ is an isometry and the same time we have established that:

for any open neighbourhood $U$ of $S_{0}$ there is an $f_{U}$ in $A$ such $\left\|f_{U}\right\| \leqslant 2, \quad f_{U \mid S_{0}} \equiv 1$ and $\left|f_{U}(s)\right| \leqslant \frac{1}{2}$ for $s \in S-U$.

Now the Theorem follows from the Bishop criterion applied to the algebra $\tilde{A}=\left\{f \in A: f_{\mid S_{0}}=\right.$ const $\}$. $/ /$

REMARKS. The theorem we have just proved is applied to the theory of small perturbations of multiplication in function algebras (see the author's paper "Perturbations of Banach algebras" to appear and also [2], [3], [5]) but one can also get another application; for example, the following theorem due to Badé and Curtis is an immediate consequence of our theorem:

THEOREM. [Bade and Curtis]. Let $A$ be a function algebra on a compact Hausdorff space $S$ and asswme $A$ is boundedly E-normal for some $\varepsilon<\frac{1}{2}$ then $A=C(S)$.

Where $A$ is called boundedly $\varepsilon$-normal if there is a constant $K$ such that for any closed disjoint subsets $E, G$ of $S$ there is an $f$ in $A$ with $\|f\| \leqslant K,|f(s)| \leqslant \varepsilon$ for $s \in E$ and $|f(s)-1| \leqslant \varepsilon$ for $s \in G$.

Proof. Assume $A \notin C(S)$ then there is a measure $\mu$ on $S$ orthogonal to $A$; let $F=\bar{F} \subset S$ be such that $\mu(F) \neq 0$, we can assume $\mu(F)=1$, and let $U$ be an open neighbourhood of $F$ with $|\mu|(U \backslash F) \leqslant \frac{1}{4}$. By our Theorem and the assumptions $F$ is a weak peak set so there is an $f$ in $A$ such that $\|f\| \leqslant 1,\left.f\right|_{F} \equiv 1$ and $|f(s)|<\frac{1}{2} \operatorname{var}(\mu)$ for $s \in S \backslash U$. We have

$$
\begin{aligned}
0 & =\left|\int_{S} f d \mu\right|=\left|\int_{F} f d \mu+\int_{U \backslash F} f d \mu+\int_{S} f d \mu\right| \\
& \geqslant 1-\frac{1}{2}-\frac{|\mu|(S-U)}{2 \operatorname{var}(\mu)}>0
\end{aligned}
$$

and this ends the proof. 


\section{References}

[1] W. Bade and P. Curtis, Jr., "Embedding theorems for commutative Banach algebras", Pacific J. Math. 18 (1966), 391-409.

[2] K. Jarosz, "Metric and algebraic perturbations of function algebras", Proc. Edinburgh Math. Soc. 26 (1983), 383-391.

[3] B. E. Johnson, "Perturbations of Banach algebras", Proc. London Math. Soc. (3) 34 (1977), 439-458.

[4] R. Larsen, Banach algebras (Pure and Applied Mathematics \#24, Marcel Dekker, Inc., New York, 1973).

[5] R. Rochberg, "Deformation of uniform algebras", Proc. London Math. Soc. (3) 39 (1979), 93-118.

Institute of Mathematics,

Warsaw University, PKiN,

00-901 Warsaw,

Poland. 